\title{
Isoflavones-Based Liposome Formulations as Anti-Aging for Skincare
}

\author{
Sophie Hollmerus ${ }^{1}$, Sakilb Yousaf ${ }^{2}$, Yamir Islam ${ }^{1}$ and Iftikhar Khan ${ }^{1 *}$ \\ ${ }^{1}$ School of Pharmacy and Biomolecular Sciences, Liverpool John Moores University, UK \\ ${ }^{2}$ School of Pharmacy and Biomedical Sciences, University of Central Lancashire, UK
}

Submission: December 21, 2017; Published: January 08, 2018

*Corresponding author: Iftikhar Khan, School of Pharmacy and Biomolecular Sciences, Liverpool John Moores University, UK, Tel: (+44) 151231

2736 Email: I.Khan@ljmu.ac.uk, iftikharkhans@yahoo.com

\begin{abstract}
Isoflavones commonly found in plants such as soya and red clover expresses many health benefits including skin healing and anti-aging properties. The capacity to counteract aging is due to isoflavones being both anti-oxidants as well as phytoestrogens, hence preventing both extrinsic as well as intrinsic aging processes. In skincare formulations their effects could be enhanced with the aid of advanced delivery systems. Isoflavones from soya bean source has successfully been incorporated into liposomes and further used in commercially available anti-aging creams. However different plants vary in isoflavones composition. Red clover isoflavones express less affinity for the estrogen receptor whilst simultaneously containing isoflavones structures that should be easier to encapsulate in liposome vehicles compared to soya bean derived isoflavones. If liposome entrapped isoflavones could successfully be obtained, the novel liposome could have a visible effect on the skin and reduce the visible adverse outcomes of aging and moreover be advantageous in terms of endocrinological safety and/or have a higher efficiency of delivering active when compared to the currently available products on the market.
\end{abstract}

Keywords: Isoflavone; Liposome; Anti-aging; Red clover

Abbreviations: APIs: Active Pharmaceutical Ingredients; MMP: Matrix Metalloproteinases; DEJ: Dermal Epidermal Junction; ROS: Reactive Oxygen Species; HPLC-MS: High Performance Liquid Chromatography-Mass Spectrometry; TEM: Transmission Electron Microscopy; W/O/W: Water-in-Oil-in-Water

\section{Introduction}

\section{Liposomes as Topical Delivery Systems}

Liposomes are a versatile and prevalent vehicle for the delivery of actives in various cosmetic products. The advantages of using liposome's in skincare formulations are the competitive penetrative capacity, bio-compatibility, non-toxicity and nonimmunogenicity of liposome's compared to alternative delivery methods. Moreover liposome's protected and preserves the entrapped active pharmaceutical ingredients (APIs) [1]. In essence, liposomes are composed of lamellas of phospholipids or sphingolipids forming a spherical structure in an aqueous solution. The fatty-acids within the lamellas are comprised of a hydrophobic head group and a hydrophilic tail group. Furthermore, the desired API (if hydrophobic in nature) can be encapsulated within the liposomal bilayers and in the internal core of liposomes (if the API is hydrophilic) and these vesicles are further transferred and released on the target surface [25]. There are numerous variations of liposomes with different properties depending on the fatty acid composition [6].
Liposomes are subcategorized by size, number of bilayers and their charge, again with possessing different properties [7]. A simple illustration of a liposome is presented in Figure 1.

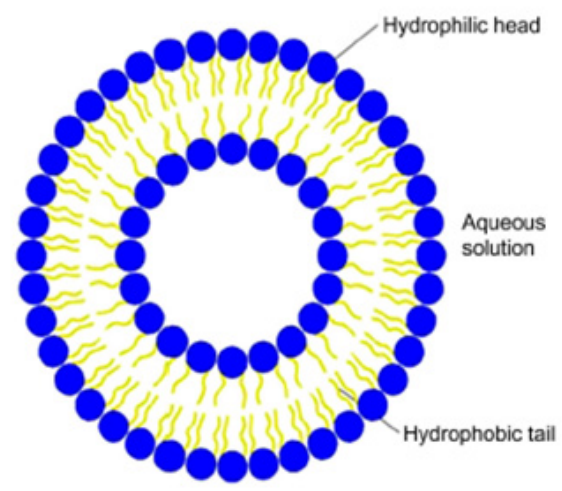

Figure 1: Structural illustration of liposome with hydrophilic and lipophilic moieties [1]. 


\section{The Aging Process}

The skins aging process is diverse and is categorized into intrinsic, extrinsic, hormonal and catabolic aging. Intrinsic aging leads to epidermal and dermal atrophy and are visibly noticeable from pale dry skin with decreased elasticity [8]. On a cellular level, increase of matrix metalloproteinases (MMP) as well as dermal epidermal junction (DEJ) is also detectable. As for extrinsic aging, the fundamental causes are UV-damage and smoking resulting in hyper pigmentation, reduced elasticity and create large deep set wrinkles. Another major cause of extrinsic aging is reactive oxygen species (ROS) which also are induced by UV-radiation [9].

The skin and its appearance are also affected by hormonal processes which are also linked to catabolic processes. For women menopause stimulates the aging of the skin as estrogen accounts for many skin benefits i.e. promotion of extracellular matrix production and maintains collagen levels, hence its decrease may have negative effects such as skin sagging [8]. One preventive method for the effect of extrinsic aging of the skin is the inhibition of the formation of ROS with the help of antioxidants [9].

\section{Isoflavones}

Trifolium Pratense also known as red clover is rich in isoflavones [10,11]. Previous research suggested that isoflavones possess many health benefits when consumed, such as prevention of cardiovascular diseases and some forms of cancer as well as calming adverse effects associated to menopause. Isoflavones have also been proven to have topical skincare benefits including anti-aging, soothing and anti-photo cancerogenic properties [12]. Isoflavones are also antioxidants [13]. Structurally isoflavones are polyphenols which occur in nature in various forms depending on their origin. As they show a close resemblance to $17-\beta$-estradiol they are also referred to as phytoestrogens [12]. The effects of phytoestrogens are controversial and there is also possible adverse health effects associated to these as they are also considered to be endocrine disruptors [14].

Isoflavones are commercially derived from a plant source, these are red clover and soya bean, however, they have some differences in their structure, red clover derived isoflavones contain more free aglycones whereas isoflavones from soy bean have a higher content of glycosidic forms. Furthermore, red clover is richer in biochanin A and formonetin [12]. Biochanin A and formonetin are precursors and are metabolized further when entering the body [14]. Chemical structures are demonstrated in Figure 2. Out of these structures, the structures more prevalent in soy, genistein and daidzin have been determined to express the most estrogenic activity and affinity to the estrogen receptor. According to research by Kato, Kajiya et al [15], the formonetin and biochanin A which are more abundant in red clover manifest a higher affinity for liposome formation in comparison to genistein and daidzein.
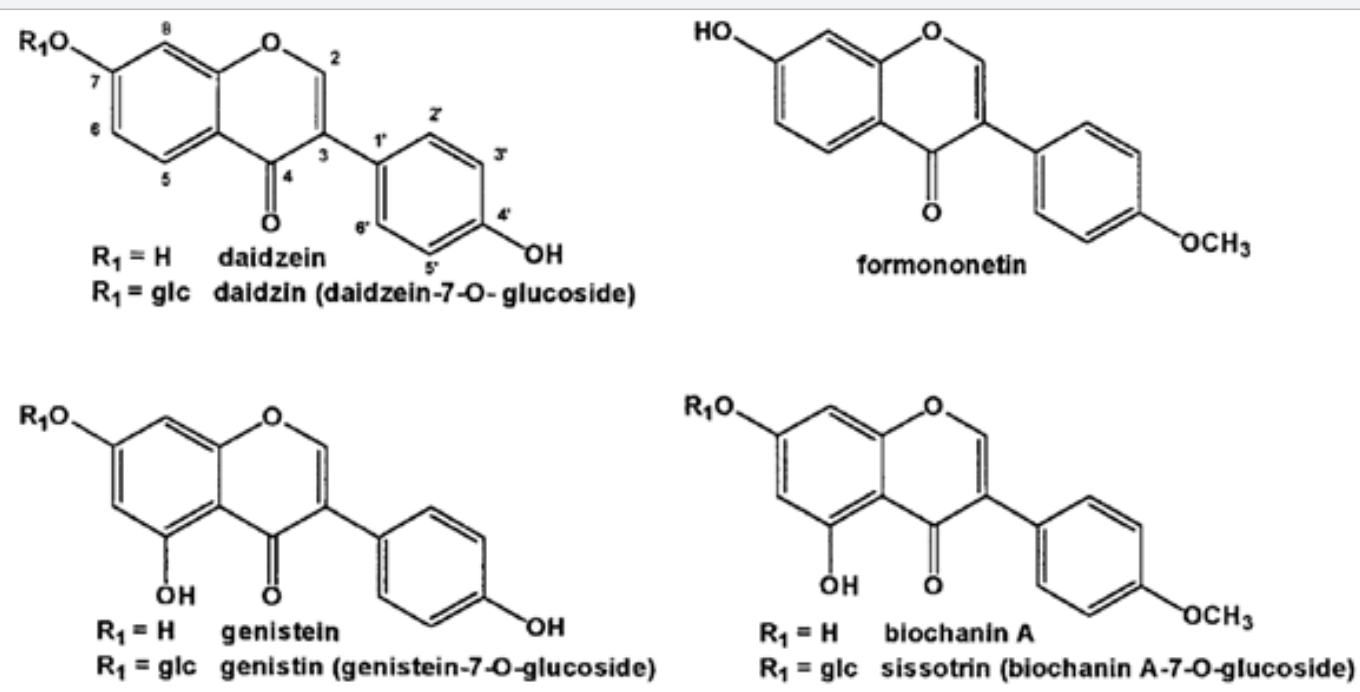

Figure 2: Chemical structures of various isoflavones. Dominant isoflavones for soy (on the left hand side) in contrast to forms prevalent in red clover (right hand side) [15].

Therefore, an incorporation of red clover extract into a liposome delivery system for topical prevention of aging is likely to be successful and result in a final product with effectively delivered antioxidant action as well as additional benefits associated to isoflavones $[16,17]$. Moreover, it could be a very gentle hormonal remedy with low risk of adverse effects for the target group of perimenopausal women. The phytoestrogenic activity on oestrogen receptors of the most prevalent isoflavones of red clover at the administered dose level in a pharmaceutics/ cosmetic formulation is assumed not to cause adverse effects and is hypothesized to interfere even less with oestrogen receptor than soy bean derived variants. Red Clover extract has already 
successfully been incorporated in the organic skincare brand. In regards to liposomes, soy bean derived isoflavones have effectively been incorporated into liposomes and further used in skincare formulations by Aveeno $\AA$ and Vichy $®$. Commercially the naturally derived active should also be of greater consumer interest than a synthetically produced equivalent [12].

\section{Techniques}

In terms of methodology there are various successful extraction techniques, however a solicited ethanol extraction is proposed as it is simple and requires few materials and instruments [18]. Despite this isoflavones have successfully been extracted from red clover for High Performance Liquid Chromatography-Mass Spectrometry (HPLC-MS) analysis with solicited solvent extraction using methanol instead of ethanol. Ethanol is proposed as a substitute to methanol due to lower toxicity $[19,20]$. Transmission electron microscopy (TEM) allows high precision imaging allowing to analyzing the morphology of the liposome identifiable, providing information regarding each individual liposome. Zeta potential and particle size analysis is a necessary compliment to the TEM analysis [21,22].

In regards to possible future cream incorporations, according to previous research isoflavonic liposomes have proven to have a higher affinity for oil in water formulation. Although aging skin typically is dehydrated oil in water systems are usually by their texture commercially unattractive [12]. In addition, research suggested that they might not have better moisturizing properties than water in oil emulsions [23]. Thus a multiple emulsion is suggested i.e. water-in-oil-in-water $(\mathrm{w} / \mathrm{o} / \mathrm{w})$ emulsion. Theoretically, this should enable the sustained release of the liposome with a more desirable texture [23]. If the proposed novel liposome is successfully produced and is clinically proven to express the hypothesized desired properties, then further research into the most suitable formulation of final product will be highly desirable for further investigation [24].

\section{Conclusion}

Isoflavones express abilities to influence processes and mechanisms known to cause visible aging of the skin. Therefore, the composition of right delivery system such as liposome using isoflavones of red clover is likely to be potent and endocrinologically safe for topical administration.

\section{Acknowledgement}

I would warmly like to thank Dr. Iftikhar Khan for mentoring, inspiration, guidance and support. Further I would also want to express my gratitude towards the advice I received from $\mathrm{Dr}$ Francesca Giuntini, Dr. Sarah Gordon and Dr. Imran Saleem

\section{References}

1. Gökçe Evren H, Yapar Evren Algın, Tanrıverdi Sakine Tuncay, Özer Özgen (2016) Nanocarriers in cosmetology. In Nanobiomaterials in Galenic Formulations and Cosmetics 10: 363-393.
2. Khan I, Elhissi A, Shah M, Alhnan MA, Waqar A (2013) Liposomebased carrier systems and devices used for pulmonary drug delivery. Woodhead Publishing Limited, UK pp. 395-443.

3. Khan I, Yousaf S, Subramanian S, Korale O, Alhnan MA, et al. (2015) Proliposome powders prepared using a slurry method for the generation of beclometasone dipropionate liposomes. International Journal of Pharmaceutics 496(2): 342-350.

4. Subramanian S, Khan I, Korale O, Alhnan MA, Ahmed W, et al. (2016) A simple approach to predict the stability of phospholipid vesicles to nebulization without performing aerosolization studies. International Journal of Pharmaceutics 502(1-2): 18-27.

5. Khan I, Yousaf S, Subramanian S, Korale O, Alhnan MA, et al. (2017) Proliposome Powders for the Generation of Liposomes: The Influence of Carbohydrate Carrier and Separation Conditions on Crystallinity and Entrapment of a Model Antiasthma Steroid. AAPS PharmSciTech 19(1): 262-274.

6. Jaafar Maalej C, Diab R, Andrieu V, Elaissari A, Fessi H (2010) Ethanol injection method for hydrophilic and lipophilic drug-loaded liposome preparation. Journal of Liposome Research 20(3): 228-243.

7. Patil, Jadhav (2014) Novel methods for liposome preparation. Chemistry and Physics of Lipids 177: 8-18.

8. Steventon K (2013) Factors of Skin Aging. Global Cosmetic Industry 181(6): 60-63.

9. Masaki H (2010) Role of antioxidants in the skin: Anti-aging effects. Journal of Dermatological Science 58(2): 85-90.

10. Budryn, Gałązka Czarnecka, Brzozowska, Grzelczyk, Mostowski, et al. (2018) Evaluation of estrogenic activity of red clover (Trifolium pratense L.) sprouts cultivated under different conditions by content of isoflavones, calorimetric study and molecular modeling. Food Chemistry 245: 324-336.

11. Clarke D, Bailey V, Lloyd A (2008) Determination of phytoestrogens in dietary supplements by LC-MS/MS. Food Additives \& Contaminants: Part A 25(5): 534-547.

12. Nemitz M, Moraes C, Koester R, Bassani C, Poser L, et al. (2015) Bioactive soy isoflavones: Extraction and purification procedures, potential dermal use and nanotechnology-based delivery systems. Phytochemistry Reviews 14(5): 849-869.

13. Tyug, Prasad, Ismail (2010) Antioxidant capacity, phenolics and isoflavones in soybean by-products. Food Chemistry 123(3): 583-589.

14. Nie Q, Xing M, Hu J, Hu X, Nie S, et al. (2017) Metabolism and health effects of phyto-estrogens. Critical Reviews in Food Science and Nutrition 57(11): 2432-2454.

15. Kato R, Kajiya K, Tokumoto H, Kumazawa S, Nakayama T (2003) Affinity of isoflavonoids for lipid bilayers evaluated with liposomal systems. BioFactors 19(3-4): 179-187.

16. Kanouni M, Rosano H, Naouli N (2002) Preparation of a stable double emulsion (W-1/0/W-2): Role of the interfacial films on the stability of the system. Advances in Colloid and Interface Science 99(3): 229-254.

17. Klejdus, Vitamvásová Štěrbová, Kubáň (2001) Identification of isoflavone conjugates in red clover (Trifolium pratense) by liquid chromatography-mass spectrometry after two-dimensional solidphase extraction. Analytica Chimica Acta 450(1-2): 81-97.

18. Liggins, Bluck, Coward, Bingham (1998) Extraction and Quantification of Daidzein and Genistein in Food. Analytical Biochemistry 264(1): 1-7.

19. Vale A (2003) Methanol. Medicine 31(10): 60-61.

20. Maier, Ovesen, Allen, York, Gadagbui, et al. (2015) Safety assessment for ethanol-based topical antiseptic use by health care workers: 
Evaluation of developmental toxicity potential. Regulatory Toxicology and Pharmacology 73(1): 248-264

21. Wang, Feng, Zheng (2016) A comparison between conventional liposome and drug-cyclodextrin complex in liposome system. International Journal of Pharmaceutics 513(1-2): 387-392.

22. Sebaaly, Jraij, Fessi, Charcosset, Greige Gerges (2015) Preparation and characterization of clove essential oil-loaded liposomes. Food Chemistry 178: 52-62.

This work is licensed under Creative Commons Attribution 4.0 License

DOI: $10.19080 / N A P D D .2018 .03 .555615$
23. Barel AO, Marc Paye, Howard I Maibach (2014) Handbook of cosmetic science and technology. Boca Raton, Boca Raton: Taylor \& amp; Francis pp. 725.

24. Betz, Aeppli, Menshutina, Leuenberger (2005) In vivo comparison of various liposome formulations for cosmetic application. International Journal of Pharmaceutics 296(1-2): 44-54.

\section{Your next submission with Juniper Publishers will reach you the below assets}

- Quality Editorial service

- Swift Peer Review

- Reprints availability

- E-prints Service

- Manuscript Podcast for convenient understanding

- Global attainment for your research

- Manuscript accessibility in different formats

( Pdf, E-pub, Full Text, Audio)

- Unceasing customer service

Track the below URL for one-step submission https://juniperpublishers.com/online-submission.php 